\title{
Silicon Phthalocyanine 4
}

National Cancer Institute

\section{Source}

National Cancer Institute. Silicon Phthalocyanine 4. NCI Thesaurus. Code C2651.

A synthetic photosensitizer agent containing a large macrocyclic ring chelated with silicon. Silicon phthalocyanine 4 localizes primarily in mitochondrial cytosolic membranes and, after photoexcitation, forms reactive oxygen species that induce apoptosis. 